TRANSACTIONS OF THE

AMERICAN MATHEMATICAL SOCIETY

Volume 359, Number 8, August 2007, Pages 3913-3931

S 0002-9947(07)04213-4

Article electronically published on March 7, 2007

\title{
COMPLEX SYMMETRIC OPERATORS AND APPLICATIONS II
}

\author{
STEPHAN RAMON GARCIA AND MIHAI PUTINAR
}

\begin{abstract}
A bounded linear operator $T$ on a complex Hilbert space $\mathcal{H}$ is called complex symmetric if $T=C T^{*} C$, where $C$ is a conjugation (an isometric, antilinear involution of $\mathcal{H}$ ). We prove that $T=C J|T|$, where $J$ is an auxiliary conjugation commuting with $|T|=\sqrt{T^{*} T}$. We consider numerous examples, including the Poincaré-Neumann singular integral (bounded) operator and the Jordan model operator (compressed shift). The decomposition $T=C J|T|$ also extends to the class of unbounded $C$-selfadjoint operators, originally introduced by Glazman. In this context, it provides a method for estimating the norms of the resolvents of certain unbounded operators.
\end{abstract}

\section{INTRODUCTION}

In his consideration of the classical Carathéodory-Fejér problem in function theory, Takagi 25] observed the relevance of the antilinear eigenvalue problem $T \mathbf{x}=\lambda \overline{\mathbf{x}}$, where $T$ is an $n \times n$ symmetric complex matrix and $\overline{\mathbf{x}}$ denotes complex conjugation of a vector $\mathbf{x}$ in $\mathbb{C}^{n}$. He noted that this equation implies that $T^{*} T \mathbf{x}=|\lambda|^{2} \mathbf{x}$ and hence that $|\lambda|$ is an eigenvalue of $|T|=\sqrt{T^{*} T}$. This observation has many consequences, for example a formula for $\|T\|$ which does not explicitly involve the computation of $|T|$ :

$$
\|T\|=\sup \left\{\sigma \geq 0:\left(\exists \mathbf{x} \in \mathbb{C}^{n}\right)((\mathbf{x} \neq \mathbf{0}) \wedge(T \mathbf{x}=\sigma \overline{\mathbf{x}}))\right\} .
$$

In this note, we consider Takagi's antilinear eigenproblem in a much more general setting.

We now pass to a separable complex Hilbert space $\mathcal{H}$ which carries a conjugation $C$. Specifically, $C$ is an antilinear operator on $\mathcal{H}$ which is involutive $\left(C^{2}=I\right)$ and isometric, meaning that $\langle x, y\rangle=\langle C y, C x\rangle$ holds for all $x, y$ in $\mathcal{H}$. A bounded operator $T: \mathcal{H} \longrightarrow \mathcal{H}$ is called $C$-symmetric if $T=C T^{*} C$ and complex symmetric if it is $C$-symmetric with respect to some conjugation $C$.

In particular, an $n \times n$ matrix $T$ is symmetric if and only if $T=C T^{*} C$ where $C$ denotes the standard conjugation $C\left(z_{1}, z_{2}, \ldots, z_{n}\right)=\left(\overline{z_{1}}, \overline{z_{2}}, \ldots, \overline{z_{n}}\right)$ on $\mathbb{C}^{n}$. Thus complex symmetric operators generalize the notion of complex symmetric matrices. In fact, $T$ is $C$-symmetric if and only if it has a symmetric matrix representation with respect to an orthonormal basis whose elements are fixed by $C$.

Received by the editors November 9, 2004 and, in revised form, July 20, 2005.

2000 Mathematics Subject Classification. Primary 30D55, 47A15.

Key words and phrases. Complex symmetric operator, Takagi factorization, inner function, Aleksandrov-Clark operator, Clark operator, Aleksandrov measure, compressed shift, Jordan operator, $J$-selfadjoint operator, Sturm-Liouville problem.

This work was partially supported by the National Science Foundation Grant DMS-0350911. 
The class of complex symmetric operators is surprisingly large. It includes all normal operators, Hankel operators, compressed Toeplitz operators (including finite Toeplitz matrices and the compressed shift), and many standard integral operators such as the Volterra operator [8. Somewhat confusingly, the unbounded analogues of $C$-symmetric operators are sometimes referred to as $J$-selfadjoint, although neither concept should be confused with the notion of $J$-selfadjointness arising in the theory of Krein spaces (where $J$ is a linear involution).

The aim of the present article is to analyze the structure of complex symmetric operators beyond Takagi's decomposition. We prove, for example, that a bounded $C$-symmetric operator $T$ factors as $T=C J|T|$, where $J$ is an auxiliary conjugation which commutes with $|T|$. This can be viewed as a generalization of a theorem of Godič and Lucenko which states that every unitary operator $U$ on $\mathcal{H}$ decomposes as the product $U=C J$ of two conjugations [12]. We use the decomposition $T=C J|T|$ to attack Takagi's antilinear eigenvalue problem in a more general setting.

Glazman pioneered the study of unbounded complex symmetric operators [10, 11] and proved that a parallel to von Neumann's theory of selfadjoint extensions of a symmetric operator exists. Specifically, one says that a closed-graph, densely defined, unbounded operator $T$ is $C$-symmetric if $T \subset C T^{*} C$ and $C$-selfadjoint if $T=C T^{*} C$. In concrete applications, $C$ is typically of the form $[C f](\mathbf{x})=$ $\overline{f(\mathbf{x})}$ or $[C f](\mathbf{x})=\overline{f(-\mathbf{x})}$ on an appropriate $L^{2}$ space. Since Glazman's time, his fundamental ideas have been applied to several classes of differential operators (see 3, 16, 20]). Moreover, the complex scaling technique, a standard tool in the theory of Schrödinger operators, naturally leads to the consideration of $C$-selfadjoint operators [9].

We show that every unbounded $C$-selfadjoint operator $T$ with zero in its resolvent admits a decomposition of the form $T=C J|T|$, where $|T|$ is positive and selfadjoint (in the usual sense) and $J$ is a conjugation strongly commuting with $|T|$. This establishes a direct connection between $C$-selfadjoint and selfadjoint operators and leads to a new method of estimating the norm of $C$-selfadjoint operators with compact resolvent.

If $T$ is an unbounded $C$-selfadjoint operator which has compact resolvent at zero, then there exists an orthonormal basis $u_{n}$ of $\mathcal{H}$ consisting of solutions to the antilinear eigenvalue problem $T f=\sigma C f$ (for $\sigma \geq 0$ ). Moreover, we have the formula

$$
\|T\|=\sup \{\sigma \geq 0:(\exists f \in \mathcal{H})((f \neq 0) \wedge(T f=\sigma C f))\} .
$$

On the other hand, the linear eigenvalue problem $T f=\lambda f$ (for $\lambda$ in $\mathbb{C}$ ) for the same operator does not in general produce an orthonormal system of eigenfunctions, nor a complete system of them (see [13, 15). Several applications of this approach, dealing with Schrödinger operators with spectral gaps and the scaled Hamiltonians appearing in the problem of resonances, can be found in [9].

The present paper consists of three distinct parts. The first section deals with the abstract structure of complex symmetric operators and briefly explores several basic examples. In the second section, we discuss Jordan model operators (compressed shifts) and their rank-one unitary perturbations. The third section is devoted to applications to unbounded operators. 


\section{Structure of COMPleX Symmetric OPERATORS}

We first review a beautiful, yet little-known, result of Godič and Lucenko (Theorem 1) on the structure of unitary operators before proving a broad generalization (Theorem 2) of their theorem to the class of all complex symmetric operators. The remainder of this section is devoted to various examples and applications.

2.1. A theorem of Godič and Lucenko. It is well-known that any planar rotation can be obtained as the product of two reflections. The following theorem of Godič and Lucenko [12] generalizes this simple geometric notion and provides an interesting perspective on the structure of unitary operators:

Theorem 1 (Godič-Lucenko). If $U$ is a unitary operator on a Hilbert space $\mathcal{H}$, then there exist conjugations $C$ and $J$ on $\mathcal{H}$ such that $U=C J$.

This theorem is remarkable, for it states that all unitary operators (on a fixed Hilbert space $\mathcal{H}$ ) can be constructed using essentially the same antilinear operator. Indeed, any conjugation on $\mathcal{H}$ can be represented as entry-by-entry complex conjugation with respect to a certain orthonormal basis (i.e. can be represented as the canonical conjugation on an appropriate $l^{2}$-space). In this sense, the conjugations $C$ and $J$ in Theorem 1 are essentially identical objects. Thus the fine structure of unitary operators arises entirely in how two copies of the same object are put together. The converse of Theorem 1 is also true:

Lemma 1. If $C$ and $J$ are conjugations on a Hilbert space $\mathcal{H}$, then $U=C J$ is a unitary operator. Moreover, $U$ is both $C$-symmetric and J-symmetric.

Proof. If $U=C J$, then (by the isometric property of $C$ and $J$ ) it follows that $\left\langle f, U^{*} g\right\rangle=\langle U f, g\rangle=\langle C J f, g\rangle=\langle C g, J f\rangle=\langle f, J C g\rangle$ for all $f, g$ in $\mathcal{H}$. Thus $U^{*}=J C$ from which $C U=U^{*} C$ and $J U=U^{*} J$ both follow.

Example 1. Let $U: \mathbb{C}^{n} \longrightarrow \mathbb{C}^{n}$ be a unitary operator with $n$ (necessarily unimodular) eigenvalues $\xi_{1}, \xi_{2}, \ldots, \xi_{n}$ and corresponding orthonormal eigenvectors $e_{1}, e_{2}, \ldots, e_{n}$. If $C$ and $J$ are defined by setting $C e_{k}=\xi_{k} e_{k}$ and $J e_{k}=e_{k}$ for $k=1,2, \ldots, n$ and extending antilinearly to all of $\mathbb{C}^{n}$, then clearly $U=C J$. By introducing offsetting unimodular parameters in the definitions of $C$ and $J$, one sees that the Godič-Lucenko decomposition of $U$ is not unique.

Example 2. If $U$ denotes the unitary operator $[U f]\left(e^{i \theta}\right)=e^{i \theta} f\left(e^{i \theta}\right)$ on $L^{2}(\partial \mathbb{D}, \mu)$, then $U=C J$ where

$$
[C f]\left(e^{i \theta}\right)=e^{\frac{i}{2} \theta} \overline{f\left(e^{i \theta}\right)}, \quad[J f]\left(e^{i \theta}\right)=e^{-\frac{i}{2} \theta} \overline{f\left(e^{i \theta}\right)}
$$

for all $f$ in $L^{2}(\partial \mathbb{D}, \mu)$. Clearly, the proof of Theorem 1 follows from the spectral theorem and this simple example.

Example 3. Let $\mathcal{H}=L^{2}(\mathbb{R}, d x)$ and let

$$
[\mathcal{F} f](\xi)=\frac{1}{\sqrt{2 \pi}} \int_{\mathbb{R}} e^{-i x \xi} f(x) d x
$$

denote the Fourier transform of a function $f$ in $L^{2}(\mathbb{R})$. Complex conjugation $[J f](x)=\overline{f(x)}$ satisfies $J \mathcal{F}^{*}=\mathcal{F} J$, whence $\mathcal{F}$ is a $J$-symmetric unitary operator. Thus $C=\mathcal{F} J$ is another conjugation operator on $L^{2}(\mathbb{R})$. The Fourier transform is thus the product of two simple conjugations: $C$ is a complex conjugation in the frequency domain and $J$ is a complex conjugation in the state space domain. 
Example 4. Let $\mathcal{H}=L^{2}(\mathbb{R}, d x)$ and let

$$
[H f](x)=p \cdot v \cdot \frac{1}{\pi i} \int_{\mathbb{R}} \frac{f(y)}{y-x} d y
$$

denote the (self-adjoint) Hilbert transform of a function $f$ in $L^{2}(\mathbb{R})$. One can verify that $H$ is $C$-symmetric with respect to the conjugation $[C f](x)=\overline{f(-x)}$ on $L^{2}(\mathbb{R})$ and that the conjugation $J$ is given by

$$
[J f](x)=p \cdot v \cdot \frac{-1}{\pi i} \int_{\mathbb{R}} \frac{\overline{f(y)}}{y+x} d y .
$$

Surprisingly, Theorem 1 has a natural generalization to the entire class of complex symmetric operators. We discuss this result below.

2.2. Refined polar decomposition of complex symmetric operators. Recall that the polar decomposition $T=U|T|$ of an operator $T$ expresses $T$ uniquely as the product of a positive operator $|T|=\sqrt{T^{*} T}$ and a partial isometry $U$ that satisfies $\operatorname{ker} T=\operatorname{ker} U=\operatorname{ker}|T|$ and that maps the initial space $(\operatorname{ker}|T|)^{\perp}$ onto the final space $\operatorname{cl}(\operatorname{ran} T)$, the closure of the range of $T$.

If $T$ is a $C$-symmetric operator, then it turns out that the partial isometry $U$ is also $C$-symmetric (for the same $C$ ). Furthermore, $U$ can be written as the product $U=C J$ of the original conjugation $C$ and a partial conjugation $J$ which commutes with $|T|$. In the case where $T$ is unitary, this decomposition reduces to the GodičLucenko decomposition for unitary operators.

To be specific, we say that an antilinear operator $J$ is a partial conjugation if $J$ restricts to a conjugation on ( $\operatorname{ker} J)^{\perp}$ (having values in the same space). In particular, the linear operator $J^{2}$ is the orthogonal projection onto the closed subspace $\operatorname{ran} J=(\operatorname{ker} J)^{\perp}$. Note that a partial conjugation $J$ can always be extended to a conjugation $\widetilde{J}$ on the entire space $\mathcal{H}$ by forming the internal direct sum $\widetilde{J}=J \oplus J^{\prime}$ where $J^{\prime}$ is any partial conjugation with support ker $J$.

Theorem 2. If $T=U|T|$ is the polar decomposition of a $C$-symmetric operator $T$, then $T=C J|T|$ where $J$ is a partial conjugation, supported on $\operatorname{cl}(\operatorname{ran}|T|)$, which commutes with $|T|=\sqrt{T^{*} T}$. In particular, the partial isometry $U$ is $C$-symmetric and factors as $U=C J$.

Proof. Write the polar decomposition $T=U|T|$ of $T$ and note that

$$
T=C T^{*} C=C|T| U^{*} C=C\left(U^{*} U\right)|T| U^{*} C=\left(C U^{*} C\right)\left(C U|T| U^{*} C\right)
$$

since $U^{*} U$ is the orthogonal projection onto $\operatorname{cl}(\operatorname{ran}|T|)$. Setting $W=C U^{*} C$, it follows that $W^{*}=C U C$ and hence $W W^{*} W=W$ since $U^{*} U U^{*}=U^{*}$. Thus $W$ is a partial isometry. Since $A=C U|T| U^{*} C$ is clearly positive, if we can show that $\operatorname{ker} A=\operatorname{ker} W=\operatorname{ker} T$, then the uniqueness of the factors in the polar decomposition of $T$ will allow us to conclude that $W=U$ and $A=|T|$.

Since $U$ and $U^{*}$ have $\operatorname{cl}(\operatorname{ran}|T|)$ as their initial and final spaces, respectively, it follows that $\operatorname{ker} W=\operatorname{ker} A=\operatorname{ker} U^{*} C$. We claim that $\operatorname{ker} T=\operatorname{ker} U^{*} C$. Clearly $\operatorname{ker} U^{*} C \subseteq \operatorname{ker} T$ by (11). Conversely, if $T f=0$, then (11) implies that $|T| U^{*} C f=0$. Since the final space of $U^{*}$ is $\operatorname{cl}(\operatorname{ran}|T|)$, we must have $U^{*} C f=0$ and hence $\operatorname{ker} T=\operatorname{ker} U^{*} C$. This proves that $U=W$ and $|T|=A$.

The equality $U=C U^{*} C$ shows that $U$ is $C$-symmetric. Writing $J=C U=U^{*} C$, we see that $J^{2}=\left(U^{*} C\right)(C U)=U^{*} U$, the orthogonal projection onto $\operatorname{cl}(\operatorname{ran}|T|)$. Since $C U|T| U^{*} C=|T|$, it follows that $J|T| J=|T|$ and hence $J|T|=|T| J$. 
From $J=C U$, it follows that $\operatorname{ker} J=\operatorname{ker} U=\operatorname{ker}|T|=(\operatorname{cl}(\operatorname{ran}|T|))^{\perp}$. Since $J=U^{*} C$, it follows that $\operatorname{ran} J=\operatorname{ran} U^{*}=\operatorname{cl}(\operatorname{ran}|T|)$. Finally, $J$ is clearly isometric on $\mathrm{cl}(\operatorname{ran}|T|)$ since $C U$ is isometric there. Thus $J$ is a partial conjugation supported on $\operatorname{cl}(\operatorname{ran}|T|)$ which commutes with $|T|$. This concludes the proof.

Theorem 2 provides a simple scheme for constructing complex symmetric operators. Fix a conjugation $C$, then select a positive bounded operator $A$ and a conjugation $J$ commuting with it. Many such $J$ exist, for they can be obtained from the spectral representation of $A$ as a multiplication operator on a direct sum of Lebesgue spaces. It is easy to verify that the $T=C J A$ is $C$-symmetric and satisfies $|T|=A$. Finally, we remark that given two conjugations $C$ and $C^{\prime}$, the map $T \mapsto C^{\prime} C T$ establishes a bijection between the class of $C$-symmetric and $C^{\prime}$ symmetric operators.

Using Theorem 2, we can also obtain several strong statements about complex symmetric operators. For instance, it turns out that the partial isometry in the polar decomposition of $T$ can always be extended to a unitary operator:

Corollary 1. If $T$ is a C-symmetric operator, then $T=W|T|$ where $W$ is a $C$-symmetric unitary operator.

Proof. If $\operatorname{cl}(\operatorname{ran}|T|)=\mathcal{H}$, then $J$ is a conjugation on all of $\mathcal{H}$ and $U=C J$ is already a $C$-symmetric unitary operator. Otherwise, write $T=C J|T|$ and extend $J$ to a conjugation $\widetilde{J}$ on all of $\mathcal{H}$ using the remarks preceding Theorem 2. By Lemma 1 . the operator $W=C \widetilde{J}$ is $C$-symmetric and unitary.

Corollary 2. If $T$ is a complex symmetric operator, then $T$ is invertible if and only if its modulus $|T|=\left(T^{*} T\right)^{1 / 2}$ is invertible.

Proof. This follows immediately from the preceding corollary.

Corollary 3. If $T$ is a complex symmetric operator, then $T^{*} T$ and $T T^{*}$ are unitarily equivalent.

Proof. If $T$ is $C$-symmetric, then write $T=C J|T|$ where, without loss of generality, we assume that $J$ is a conjugation on all of $\mathcal{H}$. Since $J$ commutes with $|T|$, it also commutes with $|T|^{2}=T^{*} T$. Therefore $C J\left(T^{*} T\right)=C T^{*} T J=T C T J=\left(T T^{*}\right) C J$. By Lemma 1, $C J$ is unitary and thus $T^{*} T$ and $T T^{*}$ are unitarily equivalent.

The unitary equivalence of $T^{*} T$ and $T T^{*}$ is necessary, but not sufficient to imply the existence of a conjugation $C$ with respect to which $T$ is $C$-symmetric. Indeed, if $T$ is any operator on a finite dimensional Hilbert space, then $\sqrt{T^{*} T}$ and $\sqrt{T T^{*}}$ are unitarily equivalent. Nevertheless, there exist operators on $\mathbb{C}^{3}$ which fail to be $C$-symmetric for any choice of a conjugation $C$ (see [8]).

In the infinite-dimensional setting, it is easily seen that the preceding three corollaries are not true without the assumption that $T$ is complex symmetric. Indeed, the unilateral shift provides immediate counterexamples to all three such assertions. Nevertheless, the unilateral shift forms the basis of the following example:

Example 5. Let $S$ denote the unilateral shift $S\left(a_{0}, a_{1}, \ldots\right)=\left(0, a_{0}, a_{1}, \ldots\right)$ on $\mathcal{H}=l^{2}(\mathbb{N})$. Both $S$ and its adjoint $S^{*}\left(a_{0}, a_{1}, \ldots\right)=\left(a_{1}, a_{2}, \ldots\right)$ commute with the canonical conjugation $C\left(a_{0}, a_{1}, \ldots\right)=\left(\overline{a_{0}}, \overline{a_{1}}, \ldots\right)$ on $\mathcal{H}$. The operator $\mathcal{T}=S^{*} \oplus S$ 
on $\mathcal{H} \oplus \mathcal{H}$ is $\mathcal{C}$-symmetric with respect to the conjugation

$$
\mathcal{C}=\left(\begin{array}{ll}
0 & C \\
C & 0
\end{array}\right)
$$

on $\mathcal{H} \oplus \mathcal{H}$, and a computation shows that

$$
\underbrace{\left(\begin{array}{cc}
S^{*} & 0 \\
0 & S
\end{array}\right)}_{\mathcal{T}}=\underbrace{\left(\begin{array}{cc}
0 & C \\
C & 0
\end{array}\right)}_{\mathcal{C}} \underbrace{\left(\begin{array}{cc}
0 & C S \\
C S^{*} & 0
\end{array}\right)}_{\mathcal{J}} \underbrace{\left(\begin{array}{ll}
P & 0 \\
0 & I
\end{array}\right)}_{|\mathcal{T}|},
$$

where $P$ denotes the orthogonal projection $P\left(a_{0}, a_{1}, a_{2}, \ldots\right)=\left(0, a_{1}, a_{2}, \ldots\right)$. In particular, the partial isometry $\mathcal{U}=\mathcal{C} \mathcal{J}$ in the polar decomposition of $\mathcal{T}$ is simply $\mathcal{T}$ itself. It is easy to check that $\mathcal{J}$ is a partial conjugation supported on $\operatorname{ran}|\mathcal{T}|$ commuting with $|\mathcal{T}|=P \oplus I$. In fact,

$$
\mathcal{J}\left(\begin{array}{l}
a_{0}, a_{1}, \ldots \\
b_{0}, b_{1}, \ldots
\end{array}\right)=\left(\begin{array}{c}
0, \overline{b_{0}}, \overline{b_{1}}, \ldots \\
\overline{a_{1}}, \overline{a_{2}}, \overline{a_{3}}, \ldots
\end{array}\right) .
$$

From here it is easy to see how to extend $\mathcal{J}$ to a conjugation $\widetilde{\mathcal{J}}$ on all of $\mathcal{H} \oplus \mathcal{H}$ :

$$
\widetilde{\mathcal{J}}\left(\begin{array}{c}
a_{0}, a_{1}, \ldots \\
b_{0}, b_{1}, \ldots
\end{array}\right)=\left(\begin{array}{c}
\overline{a_{0}}, \overline{b_{0}}, \overline{b_{1}}, \ldots \\
\overline{a_{1}}, \overline{a_{2}}, \overline{a_{3}}, \ldots
\end{array}\right) .
$$

Moreover, the operator $\mathcal{W}=\mathcal{C} \widetilde{\mathcal{J}}$ from Corollary 1 is clearly unitary:

$$
\mathcal{W}\left(\begin{array}{c}
a_{0}, a_{1}, \ldots \\
b_{0}, b_{1}, \ldots
\end{array}\right)=\left(\begin{array}{c}
a_{0}, b_{0}, b_{1}, \ldots \\
a_{1}, a_{2}, a_{3}, \ldots
\end{array}\right) .
$$

The authors thank M. Jury and W. Wogen for suggesting this example.

\section{COMPACT COMPLEX SYMMETRIC OPERATORS}

3.1. Singular value decomposition. Using the decomposition $T=C J|T|$ of Theorem 2, one can prove many results about compact $C$-symmetric operators. For instance, the following theorem shows that they have special singular-value (or Schmidt) decompositions. Without loss of generality, we consider the case $\operatorname{dim} \mathcal{H}=\infty$.

Theorem 3. Every compact $C$-symmetric operator $T$ is of the form

$$
T=\sum_{n=0}^{\infty} \sigma_{n}\left(C e_{n} \otimes e_{n}\right)
$$

where the $e_{n}$ are certain orthonormal eigenvectors of $|T|=\sqrt{T^{*} T}$ and the $\sigma_{n}$ are the nonzero eigenvalues of $|T|$, repeated according to multiplicity.

Proof. Since $T$ is compact, the mutually orthogonal eigenspaces $\mathcal{E}_{n}$ of $|T|$ corresponding to the distinct nonzero eigenvalues $\lambda_{n}$ are finite dimensional, say of dimension $d_{n}$. Let $0 \leq n<N$, where $N$ is finite if $T$ is of finite rank, or set $N=\infty$ otherwise. By Theorem 2, we may write $C T=J|T|$ where $J$ is a partial conjugation supported on $\operatorname{cl}(\operatorname{ran}|T|)$ commuting with $|T|$. In particular, $J$ restricts to a conjugation on each spectral subspace $\mathcal{E}_{n}$ of $|T|$ and hence (see [8, Lemma 1] or [1, p.94]) there exists an orthonormal basis $u_{n 1}, u_{n 2}, \ldots, u_{n d_{n}}$ for $\mathcal{E}_{n}$ which is fixed by 
$J$. In other words, we have $C T u_{n k}=\lambda_{n} u_{n k}$ for $k=1,2, \ldots, d_{n}$ which shows that the operator

$$
T-\sum_{n=0}^{N-1} \lambda_{n} \sum_{k=1}^{d_{n}}\left(C u_{n k} \otimes u_{n k}\right)
$$

vanishes on $\operatorname{cl}(\operatorname{ran}|T|)=(\operatorname{ker}|T|)^{\perp}$. Since $\operatorname{ker} T=\operatorname{ker}|T|$, it follows that (3) vanishes identically. Convergence is guaranteed since the $u_{n k}$ are orthonormal and $\lambda_{n}$ tends to 0 . The desired representation (2) follows upon a suitable relabeling of terms.

Corollary 4. If $T$ is a compact $C$-symmetric operator, then

$$
\|T\|=\sup \{\sigma \geq 0:(\exists f)((f \neq 0) \wedge(T f=\sigma C f))\} .
$$

A famous theorem of Adamyan, Arov, and Krein (AAK) states that if $T$ is a compact Hankel operator, then its singular values $\sigma_{0}, \sigma_{1}, \ldots$, repeated according to multiplicity, are given by

$$
\sigma_{n}=\inf _{\substack{\text { rank } T^{\prime}=n \\ T^{\prime} \text { Hankel }}}\left\|T-T^{\prime}\right\|
$$

An analogous theorem holds for the class of $C$-symmetric operators:

Theorem 4 ( $C$-symmetric AAK). If $T$ is a compact $C$-symmetric operator with singular values $\sigma_{0}, \sigma_{1}, \ldots$, repeated according to multiplicity, then

$$
\sigma_{n}=\inf _{T^{\prime} \stackrel{\operatorname{rank} T^{\prime}=n}{\text { C-symmetric }}}\left\|T-T^{\prime}\right\| .
$$

Proof. Write $T=C J|T|$ by Theorem 2, and using the method of proof of Theorem 3. write $|T|=\sum_{k=0}^{\infty} \sigma_{k} e_{k} \otimes e_{k}$ where $J e_{k}=e_{k}$ for all $k$. Let $A_{0}=0$ and $A_{n}=$ $\sum_{k=0}^{n-1} \sigma_{k}\left(e_{k} \otimes e_{k}\right)$ for $n \geq 1$ and note that $T^{\prime}=C J A_{n}$ satisfies

$$
\left\|T-T^{\prime}\right\|=\left\|C J|T|-C J A_{n}\right\|=\left\|C J\left(|T|-A_{n}\right)\right\|=\left\||T|-A_{n}\right\|=\sigma_{n} .
$$

The operator $T^{\prime}$ has rank $n$ and (since $J$ commutes with $A_{n}$ ) is $C$-symmetric by the comments following Theorem 2

3.2. Complex symmetric integral operators. Among the simplest examples of compact complex symmetric operators are certain integral operators. If $(X, \mu)$ is a $\sigma$-finite measure space (with $\mu$ real-valued), then a set function $\Phi$ is called a measure-preserving symmetry of $X$ if $\mu \circ \Phi=\mu$ and $\Phi^{2}=I$. With a slight abuse of notation, each measure-preserving symmetry $\Phi$ provides a conjugation on $L^{2}(X, \mu)$ via the formula $[C f](x)=\overline{f(\Phi(x))}$. The proof of the following lemma is straightforward and omitted.

Lemma 2. A bounded integral operator of the form

$$
[T f](x)=\int_{X} K(x, y) f(y) d \mu(y)
$$

on $L^{2}(X, \mu)$ is $C$-symmetric with respect to $[C f](x)=\overline{f(\Phi(x))}$ if and only if the kernel satisfies $K(\Phi x, \Phi y)=K(y, x)$ for all $x, y \in X$. 
The Volterra operator illustrates many of the concepts developed above. Moreover, it demonstrates how the $C$-symmetry of an integral operator is related to functional equations satisfied by its kernel and the measure theoretic symmetries of the underlying measure space. It also illustrates the special singular value decomposition (Theorem 3) of a compact complex symmetric operator and its relationship to the double Fourier expansion of the integral kernel. A more traditional analysis of the Volterra operator can be found in [14, Problem 188].

Example 6. Consider the Volterra integration operator

$$
[T f](x)=\int_{0}^{x} f(t) d t
$$

on $L^{2}[0,1]$, which is $C$-symmetric with respect to $[C f](x)=\overline{f(1-x)}$ (see [8]). Indeed, Lemma 2 says that we can read this directly from the functional equation $K(x, y)=K(1-y, 1-x)$ satisfied by the integral kernel, the characteristic function of the triangle $\{(x, y): 0 \leq y \leq x \leq 1\}$.

Since ker $T$ is obviously trivial, by Theorem 2 we may write $T=C J|T|$ where $J$ is a conjugation on $L^{2}[0,1]$ which commutes with $|T|$ and its spectral projections. Since each spectral subspace of $|T|$ has an orthonormal basis fixed by $J$, to diagonalize $|T|$ we consider the antilinear equation $|T| f=\sigma J f$, where $\sigma \geq 0$. In light of the decomposition $T=C J|T|$, this is equivalent to $T f=\sigma C f$ :

$$
\int_{0}^{x} f(y) d y=\sigma \overline{f(1-x)}
$$

The preceding equation yields the boundary condition $f(1)=0$. Differentiation of (44) yields $f(x)=-\sigma \overline{f^{\prime}(1-x)}$ and hence (after back-substitution)

$$
\int_{0}^{x} f(y) d y=-\sigma^{2} f^{\prime}(x)
$$

giving the second boundary condition $f^{\prime}(0)=0$. Differentiation of (5) provides the second order boundary value problem

$$
f^{\prime \prime}+\frac{1}{\sigma^{2}} f=0, \quad f(1)=0, f^{\prime}(0)=0 .
$$

Solving the boundary value problem yields $\sigma_{n}=\left[\left(n+\frac{1}{2}\right) \pi\right]^{-1}$ of $|T|$ and the associated normalized eigenfunctions $\sqrt{2} \cos \left(n+\frac{1}{2}\right) \pi x$ (where $n \geq 0$ ). To satisfy (4), we multiply these eigenfunctions by suitable unimodular constants, obtaining the unit eigenfunctions $e_{n}(x)=i^{n} \sqrt{2} \cos \left(n+\frac{1}{2}\right) \pi x$ of $|T|$, all of which are fixed by the $J$ conjugation.

Applying $C$, we obtain $\left[C e_{n}\right](x)=(-i)^{n} \sqrt{2} \sin \left(n+\frac{1}{2}\right) \pi x$ and hence (by Theorem 3) the singular value decomposition

$$
T=\sum_{n=0}^{\infty} \frac{2}{\left(n+\frac{1}{2}\right) \pi}\left[\sin \left(\pi\left(n+\frac{1}{2}\right) x\right) \otimes \cos \left(\pi\left(n+\frac{1}{2}\right) x\right)\right]
$$

of the Volterra operator. From (6) , we immediately read the numerical quantities $\|T\|=2 / \pi$ and $\operatorname{tr} T^{*} T=1 / 2$. Writing (6) explicitly, we find that

$$
[T f](x)=\int_{0}^{1}\left[\sum_{n=0}^{\infty} \frac{2}{\left(n+\frac{1}{2}\right) \pi} \sin \left(\pi\left(n+\frac{1}{2}\right) x\right) \cos \left(\pi\left(n+\frac{1}{2}\right) y\right)\right] f(y) d y,
$$

the term in brackets being a double Fourier expansion of the Volterra kernel. 
3.3. Poincaré-Neumann problem. The next example is slightly more involved, dealing with the classical two dimensional version of the double layer potential, written in complex coordinates.

In the potential theory of a simply connected planar domain $\Omega$ with piecewise smooth boundary, the operator

$$
T_{\Omega} f(z)=\frac{1}{\pi} \int_{\Omega} \frac{f(w) d A(w)}{(\bar{w}-\bar{z})^{2}}
$$

defined for $f$ in $L^{2}(\Omega, d A$ ), plays a significant role (see for instance 24]). Here $d A$ stands for area measure and the integral is taken as a Cauchy principal value. If $[C f](z)=\overline{f(z)}$ denotes complex conjugation of a function $f$ of $L^{2}(\Omega)$ (we henceforth suppress the $d A$ ), then clearly $T_{\Omega}$ is a $C$-symmetric operator.

The case $\Omega=\mathbb{C}$ is particularly important. Some simple manipulations with single and double layer potentials carried by $\partial \Omega$ (or any closed curve) reveal that $C T_{\mathbb{C}}$ is a conjugation on $L^{2}(\mathbb{C})$ 24. Returning to our formalism, we infer that $T_{\mathbb{C}}^{*} T_{\mathbb{C}}=C T_{\mathbb{C}} C T_{\mathbb{C}}=I$. In other words, $T_{\mathbb{C}}$ is a $C$-symmetric unitary operator. We may therefore write $T_{\mathbb{C}}=C J$, where the conjugation

$$
[J f](z)=\frac{1}{\pi} \int_{\mathbb{C}} \frac{\overline{f(w)} d A(w)}{(w-z)^{2}}
$$

on $L^{2}(\mathbb{C})$ is called by Schiffer the Hilbert transform of $f$.

In general, $T_{\Omega}$ is a compression of $T_{\mathbb{C}}$ to the subspace $L^{2}(\Omega)$ of $L^{2}(\mathbb{C})$. Indeed, if $P_{\Omega}$ denotes the orthogonal projection from $L^{2}(\mathbb{C})$ onto $L^{2}(\Omega)$ :

$$
P_{\Omega} f(z)= \begin{cases}f(z), & z \in \Omega, \\ 0, & z \in \mathbb{C} / \Omega,\end{cases}
$$

then $T_{\Omega}=P_{\Omega} T_{\mathbb{C}} P_{\Omega}$ (with a slight abuse of notation). Moreover, the commutativity of $C$ and $P_{\Omega}$ implies that $T_{\Omega}$ is a $C$-symmetric operator.

Let $L_{a}^{2}(\Omega)$ denote the Bergman space of $\Omega$, the subspace of all holomorphic functions in $L^{2}(\Omega)$, and let $P$ denote the orthogonal projection of $L^{2}(\Omega)$ onto $L_{a}^{2}(\Omega)$, otherwise known as the Bergman projection. A short computation shows that $P^{\prime}=$ $C P C$ is the orthogonal projection onto the subspace $C L_{a}^{2}(\Omega)$ which consists of all anti-analytic functions in $L^{2}(\Omega)$.

It turns out that the operator $T=P^{\prime} T_{\Omega} P$, which one can regard as an operator from $L_{a}^{2}(\Omega)$ to $C L_{a}^{2}(\Omega)$, is $C$-symmetric:

$$
C T=C\left(P^{\prime} T_{\Omega} P\right)=C(C P C) T_{\Omega} P=P C T_{\Omega} P=P T_{\Omega}^{*} C P=\left(P T_{\Omega}^{*} P^{\prime}\right) C=T^{*} C .
$$

Using the $C$-symmetry of $P^{\prime} T_{\Omega} P$, we obtain the following Hilbert variant of a series of observations due to Bergman and Schiffer:

Theorem 5. If $\Omega$ is a bounded planar domain with $C^{2}$ boundary, then there exists an orthonormal basis $\left(u_{n}\right)_{n=0}^{\infty}$ of the Bergman space $L_{a}^{2}(\Omega)$ and a sequence $\left(\sigma_{n}\right)_{n=0}^{\infty}$ of positive numbers such that:

$$
\frac{1}{\pi} \int_{\Omega} \frac{u_{n}(w) d A(w)}{(\bar{w}-\bar{z})^{2}}=\sigma_{n} \overline{u_{n}(z)}
$$

for all $z$ in $\Omega$. 
Proof. The operator $T_{\Omega}$ is compact (see [2], specifically the analysis of the $L$-kernel) and hence so is $T=P^{\prime} T_{\Omega} P$. Since $T$ is supported on $L_{a}^{2}(\Omega)$, the result follows from Theorem 3 ,

The values $\sigma_{n}$ for which (7) is solvable are known as the Fredholm eigenvalues of $\Omega$, and the associated eigenfunctions $u_{n}$ (canonically attached by (7) to any bounded planar domain) are remarkable in many respects. For instance they simultaneously diagonalize the Bergman kernel $K_{\Omega}$ and the $L$-kernel $L_{\Omega}$ of the domain:

$$
K_{\Omega}(z, w)=-\frac{2}{\pi} \frac{\partial^{2} G(z, w)}{\partial z \partial \bar{w}}=\sum_{n=0}^{\infty} u_{n}(z) \overline{u_{n}(w)}, \quad z, w \in \Omega
$$

and

$$
L_{\Omega}(z, w)=-\frac{2}{\pi} \frac{\partial^{2} G(z, w)}{\partial z \partial w}=\sum_{n=0}^{\infty} \sigma_{n} u_{n}(z) u_{n}(w), \quad z, w \in \Omega,
$$

where $G(z, w)$ is the Green function of $\Omega$ (see [2, 24]).

\section{Jordan OPERATORS AND Clark PERTURBations}

As an extended example, we briefly discuss the decompositions $T=C J|T|$ (of Theorem 2) for the standard Jordan model operators and consider the GodičLucenko decompositions (Theorem11) of their rank-one unitary perturbations. Complete details, including all computations, can be found in [7.

4.1. Compressed Toeplitz operators. We work here in the Hardy space $H^{2}$ of the unit disk $\mathbb{D}$, and we freely identify functions in $H^{2}$ with their nontangential boundary values which exist a.e. on the unit circle $\partial \mathbb{D}$. Most of the following preliminary material can be found in the general texts [5, 17] or the more specialized book [18].

Our interest lies in the so-called model spaces $H^{2} \ominus \varphi H^{2}$, where $\varphi$ denotes a nonconstant inner function. There is a natural interplay between function theory and operator theory on the spaces $H^{2} \ominus \varphi H^{2}$, for they are examples of reproducing kernel Hilbert spaces. Indeed, it is not hard to derive from the standard properties of the Szegö kernel $e_{\lambda}(z)=(1-\bar{\lambda} z)^{-1}$ and the definition of $H^{2} \ominus \varphi H^{2}$ that the formula $f(\lambda)=\left\langle f, K_{\lambda}\right\rangle$ holds for every $f$ in $H^{2} \ominus \varphi H^{2}$. Here $K_{\lambda}$ denotes the reproducing kernel

$$
K_{\lambda}(z)=\frac{1-\overline{\varphi(\lambda)} \varphi(z)}{1-\bar{\lambda} z}
$$

for $H^{2} \ominus \varphi H^{2}$.

Recall that the Toeplitz operator with symbol $u$ in $L^{\infty}(\partial \mathbb{D})$ is the operator $T_{u}$ : $H^{2} \longrightarrow H^{2}$ defined by $T_{u} f=P(u f)$ where $P$ denotes the orthogonal projection from $L^{2}$ onto $H^{2}$. Also recall that the adjoint of a Toeplitz operator is given by the simple formula $T_{u}^{*}=T_{\bar{u}}$.

A compressed Toeplitz operator is an operator of the form $P_{\varphi} T_{u} P_{\varphi}$ where $T_{u}$ is a standard Toeplitz operator and $P_{\varphi}$ denotes the orthogonal projection from $H^{2}$ onto $H^{2} \ominus \varphi H^{2}$. With a slight abuse of notation, we will regard compressed Toeplitz operators as operators acting on the space $H^{2} \ominus \varphi H^{2}$, rather than $H^{2}$ itself. It turns out that compressed Toeplitz operators are complex symmetric operators 
with respect to the conjugation

$$
[C f](z)=\overline{f z} \varphi
$$

on $H^{2} \ominus \varphi H^{2}[7,8$ :

Theorem. If $\varphi$ is a nonconstant inner function and $u$ belongs to $L^{\infty}(\partial \mathbb{D})$, then the compressed Toeplitz operator $P_{\varphi} T_{u} P_{\varphi}$ is $C$-symmetric with respect to the conjugation (9) on $H^{2} \ominus \varphi H^{2}$.

Although $\overline{f z} \varphi$ does not at first appear to be the boundary function of an analytic function, let alone one in $H^{2} \ominus \varphi H^{2}$, it is not hard to verify. Indeed, it suffices to check that both $\langle C f, \overline{z h}\rangle$ and $\langle C f, \varphi h\rangle$ vanish whenever $f$ belongs to $H^{2} \ominus \varphi H^{2}$ and $h$ belongs to $H^{2}$.

4.2. Refined polar decomposition of compressed shifts. In this section we obtain the refined polar decomposition for the compressed shift (or Jordan operator) guaranteed by Theorem 2, In fact, we are able to consider a slight generalization of the Jordan model operator with little additional effort.

In our computations, we will make frequent use of disk automorphisms and we adopt the following notation. For each $w$ in $\mathbb{D}$, we let $b_{w}$ denote the function

$$
b_{w}(z)=\frac{z-w}{1-\bar{w} z} .
$$

We also require the reproducing kernels $K_{w}$ (8) and their conjugates under (9):

$$
\left[C K_{w}\right](z)=\frac{\varphi(z)-\varphi(w)}{z-w} .
$$

Furthermore, we frequently refer to the normalized kernel functions $k_{w}=K_{w} /\left\|K_{w}\right\|$.

For each $\lambda$ in $\mathbb{D}$, we consider the compression

$$
S_{\lambda} f=P_{\varphi}\left(b_{\lambda} f\right)
$$

of the analytic Toeplitz operator $T_{b_{\lambda}}$ to $\mathcal{H}_{\varphi}$. The operators $S_{\lambda}$ are simple generalizations of the compressed shift $S_{0} f=P_{\varphi}(z f)$. We also remark that $S_{\lambda}^{*} f=P_{\varphi}\left(\overline{b_{\lambda}} f\right)$ and that the operators $S_{\lambda}$ are $C$-symmetric with respect to (9).

Our aim in this section is to explicitly describe the factorization $S_{\lambda}=C J_{\lambda}\left|S_{\lambda}\right|$ of these operators. We first require several computational lemmas, the first of which generalizes [4, Lem. 2.1]. Detailed proofs can be found in [7.

Lemma 3. $S_{\lambda}^{*} f=f / b_{\lambda}$ if and only if $f$ is orthogonal to $k_{\lambda} . S_{\lambda} f=b_{\lambda} f$ if and only if $f$ is orthogonal to $C k_{\lambda}$.

To find the modulus $\left|S_{\lambda}\right|$ of $S_{\lambda}$, we need only describe the positive operator $S_{\lambda}^{*} S_{\lambda}$. By Lemma 3, it follows that if $f$ is orthogonal to $C k_{\lambda}$, then $S_{\lambda}^{*} S_{\lambda} f=S_{\lambda}^{*}\left(b_{\lambda} f\right)=f$. Hence $\left|S_{\lambda}\right|$ restricts to the identity operator on the orthocomplement of the onedimensional subspace spanned by the function $C k_{\lambda}$. This tells us, for example, that $\left|S_{\lambda}\right|$ maps the function $C k_{\lambda}$ onto a nonnegative constant multiple of itself. In fact:

Lemma 4. $S_{\lambda} C k_{\lambda}=-\varphi(\lambda) k_{\lambda}$ and hence $\left|S_{\lambda}\right| C k_{\lambda}=|\varphi(\lambda)| C k_{\lambda}$.

Summing up, the modulus $\left|S_{\lambda}\right|$ of $S_{\lambda}$ is given by:

$$
\left|S_{\lambda}\right|=\left[I-\left(C k_{\lambda} \otimes C k_{\lambda}\right)\right]+|\varphi(\lambda)|\left(C k_{\lambda} \otimes C k_{\lambda}\right) .
$$

In light of (13) and Lemma 3. we assume that $\varphi(\lambda) \neq 0$ since otherwise the polar decomposition of $S_{\lambda}$ is already evident. Indeed, if $\varphi(\lambda)=0$, then $\operatorname{ker} S_{\lambda}$ equals the 
one-dimensional subspace spanned by $C k_{\lambda}$ and the operator $S_{\lambda}$ acts isometrically (multiplication by $b_{\lambda}$ ) on the orthocomplement of this subspace.

By Theorem 2, we may write $S_{\lambda}=C J_{\lambda}\left|S_{\lambda}\right|$ where $J_{\lambda}$ is a partial conjugation supported on $\mathrm{cl}\left(\operatorname{ran}\left|S_{\lambda}\right|\right)=H^{2} \ominus \varphi H^{2}$ which commutes with $\left|S_{\lambda}\right|$. In particular, we see that the assumption that $\varphi(\lambda) \neq 0$ implies that $J_{\lambda}$ is a conjugation on all of $H^{2} \ominus \varphi H^{2}$. To find $J_{\lambda}$, we write

$$
J_{\lambda}\left|S_{\lambda}\right|=C S_{\lambda}
$$

and compute the action of $J_{\lambda}$ on the spectral subspaces of $\left|S_{\lambda}\right|$.

If $f$ is orthogonal to $C k_{\lambda}$, then $\left|S_{\lambda}\right| f=f$ by (13) and hence $J_{\lambda} f=C S_{\lambda} f=$ $C\left(b_{\lambda} f\right)$ by (14) and Lemma 3. Since $\varphi(\lambda) \neq 0$ we have

$$
|\varphi(\lambda)| J\left(C k_{\lambda}\right)=J\left|S_{\lambda}\right|\left(C k_{\lambda}\right)=C\left(S_{\lambda} C k_{\lambda}\right)=-\overline{\varphi(\lambda)} C k_{\lambda},
$$

the two equalities following from (14) and Lemma 4, respectively. Putting these calculations together, we have the following explicit formula for $J_{\lambda}$ :

$$
J_{\lambda} f= \begin{cases}C\left(b_{\lambda} f\right), & f \perp C k_{\lambda}, \\ \bar{\alpha} C k_{\lambda}, & f=C k_{\lambda}\end{cases}
$$

where $\alpha=-\varphi(\lambda) /|\varphi(\lambda)|$.

We can now compute the partial isometry $U_{\lambda}=C J_{\lambda}$ in the polar decomposition of $S_{\lambda}$ using (15). By our assumption that $\varphi(\lambda) \neq 0, U_{\lambda}$ is actually unitary, since $C$ and $J_{\lambda}$ are both conjugations on $H^{2} \ominus \varphi H^{2}$. Applying $C$ to (15) yields

$$
U_{\lambda} f= \begin{cases}b_{\lambda} f, & f \perp C k_{\lambda}, \\ \alpha k_{\lambda}, & f=C k_{\lambda},\end{cases}
$$

and hence (using Lemma 3) $U_{\lambda}$ is given by the formula

$$
U_{\lambda}=S_{\lambda}\left[I-\left(C k_{\lambda} \otimes C k_{\lambda}\right)\right]+\alpha\left(k_{\lambda} \otimes C k_{\lambda}\right) .
$$

We can see directly that $U_{\lambda}$ is $C$-symmetric, for a short computation shows that $U_{\lambda}$ is a rank-one $C$-symmetric unitary perturbation of $S_{\lambda}$ :

$$
U_{\lambda}=S_{\lambda}+(\alpha+\varphi(\lambda))\left(k_{\lambda} \otimes C k_{\lambda}\right) .
$$

We summarize our results in the following theorem:

Theorem 6. Let $\varphi$ be a nonconstant inner function and let $\lambda$ be a point in $\mathbb{D}$ such that $\varphi(\lambda) \neq 0$. The polar decomposition of the compressed Toeplitz operator $S_{\lambda} f=P_{\varphi}\left(b_{\lambda} f\right)$ is given by $S_{\lambda}=U_{\lambda}\left|S_{\lambda}\right|$ where $U_{\lambda}$ is the C-symmetric unitary operator (17) and $\left|S_{\lambda}\right|$ is given by (13). Moreover, $U_{\lambda}=C J_{\lambda}$ where the conjugation $J_{\lambda}$ is given by (15).

4.3. Generalized Aleksandrov-Clark operators. The operator $U_{\lambda}$ defined by (17) is not the only rank-one $C$-symmetric unitary perturbation of $S_{\lambda}$. Indeed, for any unimodular constant $\alpha$, the operator

$$
U_{\lambda, \alpha}=S_{\lambda}+(\alpha+\varphi(\lambda))\left(k_{\lambda} \otimes C k_{\lambda}\right)
$$

is $C$-symmetric and unitary, regardless of whether the inner function $\varphi$ vanishes at $\lambda$. This can be seen by expressing $U_{\lambda, \alpha}$ in a form analogous to (16) and applying the lemmas of the preceding section.

We refer to operators of the form (18) as generalized Aleksandrov-Clark operators due to their similarity to the operators considered by Clark in [4] and later by A. B. 
Aleksandrov and others (see 19 for background). Observe that each $U_{\lambda, \alpha}$ has the Godič-Lucenko decomposition

$$
U_{\lambda, \alpha}=C J_{\lambda, \alpha}
$$

where the conjugation $J_{\lambda, \alpha}$ is given by

$$
J_{\lambda, \alpha} f= \begin{cases}C\left(b_{\lambda} f\right), & f \perp C k_{\lambda}, \\ \bar{\alpha} C k_{\lambda}, & f=C k_{\lambda},\end{cases}
$$

the parameter $\alpha$ now being allowed to vary over the unit circle. This decomposition makes it easy to compute the eigenvalues and eigenvectors (if any) of each $U_{\lambda, \alpha}$.

A function $f$ is an eigenvector of $U_{\lambda, \alpha}$ corresponding to the (necessarily unimodular) eigenvalue $\xi$ if and only if

$$
J_{\lambda, \alpha} f=\bar{\xi} C f .
$$

In light of the explicit formula (19) for $J_{\lambda, \alpha}$, we take the orthogonal decomposition of $f$ with respect to the one-dimensional subspace spanned by $C k_{\lambda}$. After possibly multiplying by a constant, we may assume that $f$ is of the form $f=g+C K_{\lambda}$ where $g$ is orthogonal to $C K_{\lambda}$. Substituting this into (20) we deduce that

$$
J_{\lambda}\left(g+C K_{\lambda}\right)=\bar{\xi}\left(C g+K_{\lambda}\right) .
$$

By (19), this can be written

$$
C\left(b_{\lambda} g\right)+\bar{\alpha} C K_{\lambda}=\bar{\xi} C g+\bar{\xi} K_{\lambda} .
$$

Applying $C$ to the equation and solving for $g$ gives us

$$
g=\frac{\xi C K_{\lambda}-\alpha K_{\lambda}}{b_{\lambda}-\xi}
$$

Using the explicit formulas (8) and (11) for $K_{\lambda}$ and $C k_{\lambda}$ we find (see [7] for the explicit computations) that $f$ is a constant multiple of the function

$$
f_{\xi}(z):=\frac{1-\overline{b_{-\varphi(\lambda)}(\alpha)} \varphi(z)}{1-\overline{b_{-\lambda}(\xi)} z}
$$

where $b_{w}$ denotes the generic disk automorphism (10). Conversely, we see that if $\xi$ is a unimodular constant such that $f_{\xi}$ belongs to $H^{2}$, then $f_{\xi}$ is an eigenvector of $U_{\lambda, \alpha}$ corresponding to the eigenvalue $\xi$. Moreover, the computation above shows that the eigenspaces of $U_{\lambda, \alpha}$ are one-dimensional.

A necessary condition for a function of the form (21) to belong to $H^{2}$ is that $\varphi$ have the nontangential limiting value $b_{-\varphi(\lambda)}(\alpha)$ at the point $b_{-\lambda}(\xi)$. In other words, the condition

$$
\varphi\left(\frac{\xi+\lambda}{1+\bar{\lambda} \xi}\right)=\frac{\alpha+\varphi(\lambda)}{1+\overline{\varphi(\lambda)} \alpha}
$$

is necessary for $f_{\xi}$ to be an eigenvector of $U_{\lambda, \alpha}$ corresponding to the eigenvalue $\xi$. In general, this condition is not sufficient and we must examine the angular derivative (most easily via the local Dirichlet integral [22]) of $\varphi$ at the point $b_{-\lambda}(\xi)$. We do not wish to pursue the function theoretic details here and simply remark that (22) generalizes [4, Thm. 3.2]. 
The following lemma shows that we may select a unit vector, fixed by $C$, from each of the (necessarily one-dimensional) eigenspaces of $U_{\lambda, \alpha}$ :

Lemma 5. If $T$ is a normal $C$-symmetric operator, then the eigenspaces of $T$ are fixed by $C$.

Proof. By normality, $T f=\lambda f$ implies that $T^{*} f=\bar{\lambda} f$. Applying $C$ to the preceding gives $T(C f)=\lambda(C f)$ and thus the eigenspaces of $T$ are invariant under $C$.

In summary, if $\lambda$ and $\alpha$ are values (in $\mathbb{D}$ and on $\partial \mathbb{D}$, respectively) such that the operator $U_{\lambda, \alpha}$ has a pure point spectrum, then we can construct an orthonormal basis of $H^{2} \ominus \varphi H^{2}$ consisting of self-conjugate vectors. In particular, the matrix representation of any $C$-symmetric operator with respect to such a basis will be symmetric. Conditions which ensure that $U_{\lambda, \alpha}$ has a pure point spectrum can be obtained by suitably generalizing several theorems in 4 .

\section{UNBOUNDED COMPLEX SYMMETRIC OPERATORS}

Let $T: \mathcal{D}(T) \longrightarrow \mathcal{H}$ be a closed graph, densely defined linear operator acting on a complex Hilbert space $\mathcal{H}$ and let $C$ be a conjugation on $\mathcal{H}$. Such an operator is called $C$-symmetric if $T \subset C T^{*} C$ or, equivalently, if

$$
\langle C T f, g\rangle=\langle C T g, f\rangle
$$

for all $f, g$ in $\mathcal{D}(T)$. We say that an operator $T$ is $C$-selfadjoint if $T=C T^{*} C$ (in particular, a bounded $C$-symmetric operator is $C$-selfadjoint). Unbounded $C$ selfadjoint operators are sometimes called $J$-selfadjoint, although this should not be confused with the notion of $J$-selfadjointness in the theory of Krein spaces.

In contrast to the classical extension theory of von Neumann, it turns out that a $C$-symmetric operator always has a $C$-selfadjoint extension 10, 11] (see also [6, 20]). Indeed, the maximal antilinear symmetric operators $S$ (in the sense that $\langle S f, g\rangle=\langle S g, f\rangle$ for all $f, g$ in $\mathcal{D}(S))$ produce $C$-selfadjoint operators $C S$. Because of this, we use the term complex symmetric operator freely in both the bounded and unbounded situations when we are not explicit about the conjugation $C$. Much of this theory was developed by Glazman, whose early book [11] remains unsurpassed for its depth and elegance.

In concrete applications, $C$ is typically derived from complex conjugation on an appropriate $L^{2}$ space over a domain in $\mathbb{R}^{n}$ and $T$ is a particular non-selfadjoint differential operator. For instance, the articles [16, 20] contain a careful analysis and parametrization of boundary conditions for Sturm-Liouville type operators with complex potentials which define $C$-selfadjoint operators. Such operators also arise in studies related to Dirac-type operators [3]. The complex scaling technique, a standard tool in the theory of Schrödinger operators, also leads to the consideration of $C$-selfadjoint operators [9] and the related class of $C$-unitary operators [23].

A useful criterion for $C$-selfadjointness can be deduced from the equality

$$
\mathcal{D}\left(C T^{*} C\right)=\mathcal{D}(T) \oplus\left\{f \in \mathcal{D}\left(T^{*} C T^{*} C\right): T^{*} C T^{*} C f+f=0\right\}
$$

(see [20]). A different criterion goes back to Zhikhar [26]: if the $C$-symmetric operator $T$ satisfies $\mathcal{H}=(T-z I) \mathcal{D}(T)$ for some complex number $z$, then $T$ is $C$-selfadjoint. The resolvent set of $T$ consists of exactly the points $z$ fulfilling the latter condition. We denote the inverse to the right by $(T-z I)^{-1}$ and note that it is a bounded linear operator defined on all of $\mathcal{H}$. 
5.1. Antilinear eigenfunction expansions. Unlike their selfadjoint counterparts, unbounded $C$-selfadjoint operators do not, in general, possess a spectral resolution and fine functional calculus. Nevertheless, when an unbounded $C$-selfadjoint operator has a compact resolvent, a canonically associated antilinear eigenvalue problem always has a complete set of mutually orthogonal eigenfunctions.

Theorem 7. If $T: \mathcal{D} \longrightarrow \mathcal{H}$ is an unbounded $C$-selfadjoint operator with compact resolvent $(T-z I)^{-1}$ for some complex number $z$, then there exists an orthonormal basis $u_{0}, u_{1}, \ldots$ of $\mathcal{H}$ consisting of solutions of the antilinear eigenvalue problem:

$$
(T-z I) u_{n}=\sigma_{n} C u_{n}
$$

where $\sigma_{0}, \sigma_{1}, \ldots$ is an increasing sequence of positive numbers tending to $\infty$.

Proof. For $f, g$ in $\mathcal{D}(T)$ we have $\langle C(T-z I) f, g\rangle=\langle C(T-z I) g, f\rangle$. Let $S$ denote the compact operator $(T-z I)^{-1}$ and let $f=S x$ and $g=S y$. Since $\langle C x, S y\rangle=\langle C y, S x\rangle$ for all $x, y, S$ is a compact $C$-symmetric operator. By Theorem 3 there exists an orthonormal basis $u_{n}$ of $\mathcal{H}$ such that $S C u_{n}=\sigma_{n}^{-1} u_{n}$ for all $n$, where $\sigma_{n}^{-1}$ is a decreasing sequence of positive numbers tending to zero. Since each $u_{n}$ belongs to $\operatorname{ran} A=\mathcal{D}(T)$, we apply $T-z I$ to both sides of the preceding equation and the desired result follows.

We note several useful corollaries of the preceding theorem:

Corollary 5. If $T: \mathcal{D} \longrightarrow \mathcal{H}$ is an unbounded $C$-selfadjoint operator with compact resolvent at $z=0$, then a vector $f=\sum_{n=0}^{\infty} a_{n} u_{n}$ in $\mathcal{H}$ belongs to $\mathcal{D}(T)$ if and only if $\sum_{n=0}^{\infty} \sigma_{n}^{2}\left|a_{n}\right|^{2}<\infty$.

Corollary 6. Under the conditions of Theorem 7 ,

$$
\left\|(T-z I)^{-1}\right\|=\frac{1}{\sigma_{0}} .
$$

In the spirit of Theorem 7 we have the following $C$-selfadjointness criterion:

Theorem 8. Let $T: \mathcal{D}(T) \longrightarrow \mathcal{H}$ be a closed, densely defined, $C$-symmetric operator. If there exists a complete system of vectors $u_{n}$ in $\mathcal{D}(T)$ and an increasing positive sequence $\sigma_{n}$ tending to infinity satisfying $T u_{n}=\sigma_{n} C u_{n}$ for all $n$, then $T$ is $C$-selfadjoint.

Proof. Since $T$ is $C$-symmetric, $\sigma_{j}\left\langle u_{j}, u_{k}\right\rangle=\left\langle C T u_{j}, u_{k}\right\rangle=\left\langle C T u_{k}, u_{j}\right\rangle=\sigma_{k}\left\langle u_{k}, u_{j}\right\rangle$ and hence $u_{j} \perp u_{k}$ whenever $\sigma_{j} \neq \sigma_{k}$. In the case of higher multiplicities, say $\sigma_{n}=\sigma_{n+1}=\cdots=\sigma_{n+p}$, we may assume that the vectors $u_{n}, \ldots, u_{n+p}$ are mutually orthogonal. Indeed, if these vectors were not orthogonal, we could simply replace them with an orthonormal basis for the real vector space generated by $u_{n}, \ldots, u_{n+p}$. We can therefore assume that $u_{n}$, and hence $C u_{n}$, form orthonormal bases of $\mathcal{H}$.

Let $f=\sum_{j=1}^{\infty} a_{j} C u_{j}$ represent an arbitrary vector in $\mathcal{H}$. For each finite $n$, the vector $f_{n}=\sum_{j=1}^{n} a_{j} \sigma_{j}^{-1} u_{j}$ belongs to $\mathcal{D}(T)$ by Corollary [5 and satisfies $T f_{n}=$ $\sum_{j=1}^{n} a_{j} C u_{j}$. Since the graph of $T$ is closed, it is not hard to see that $T: \mathcal{D}(T) \longrightarrow$ $\mathcal{H}$ is surjective. According to the criterion of [26], $T$ is $C$-selfadjoint.

5.2. Refined polar decomposition of $C$-selfadjoint operators. We can generalize the refined polar decomposition $T=C J|T|$ of Theorem 2 to the case of unbounded $C$-selfadjoint operators, modulo several minor modifications. 
Theorem 9. If $T$ is a $C$-selfadjoint operator with zero in its resolvent, then $T=C J|T|$ where $|T|$ is a positive operator (in the von Neumann sense) satisfying $\mathcal{D}(|T|)=\mathcal{D}(T)$ and $J$ is a conjugation on $\mathcal{H}$ which strongly commutes with $|T|$. Conversely, any operator of the form described above is $C$-selfadjoint.

Proof. If $T$ is a $C$-selfadjoint operator with zero in its resolvent, then $T: \mathcal{D}(T) \longrightarrow$ $\mathcal{H}$ is surjective and we let $R: \mathcal{H} \longrightarrow \mathcal{H}$ denote the bounded right inverse (the resolvent at 0 ) of $T$. Since $R \mathcal{H}=\mathcal{D}(T)$, we use the fact that $T R=I$ and (23) to conclude that $\langle C f, R g\rangle=\langle C T R f, R g\rangle=\langle C T R g, R f\rangle=\langle C g, R f\rangle$ for all $f, g$ in $\mathcal{H}$. This implies that $\left\langle R^{*} C f, g\right\rangle=\langle C R f, g\rangle$ for all $f, g$ in $\mathcal{H}$ and hence $R$ is a bounded $C$-symmetric operator. In particular, $R^{*}$ is a bounded $C$-symmetric operator that is injective and has dense range.

Let $R^{*}=C J\left|R^{*}\right|$ be the decomposition of $R^{*}$ guaranteed by Theorem 2, where without loss of generality we assume $J$ is a conjugation on all of $\mathcal{H}$ which commutes with $\left|R^{*}\right|$. Taking the adjoint of this equation and substituting it into the equation $T R=I$, we see that $T\left|R^{*}\right| J C=I$ and hence $T\left|R^{*}\right|=C J$. We read from here that $\left|R^{*}\right| \mathcal{H}=\mathcal{D}(T)$ and hence the unbounded positive operator $\left|R^{*}\right|^{-1}$ has the same domain as $T$. This implies that $J C T\left|R^{*}\right|=I$, or equivalently, $J C T=\left|R^{*}\right|^{-1}$ as unbounded operators. This yields the decomposition $T=C J|T|$ where the positive self-adjoint operator $|T|$ is defined to be $\left|R^{*}\right|^{-1}$.

Regarding the terminology of Theorem 9, we say that $J$ strongly commutes with $|T|$ if $J$ commutes with the spectral measure of $|T|$. Equivalently, we could say that $J$ commutes with the bounded selfadjoint operator $|T|^{-1}$. Also observe that the operator $U=C J$ in Theorem 9 is a unitary $C$-symmetric operator.

Although we do not pursue this direction further in the present article, we remark that Theorem 9 can be used to characterize the $C$-selfadjoint extensions of an arbitrary $C$-symmetric operator.

Example 7. Using the techniques above, we briefly discuss a simple example of a first order differential operator with a nonselfadjoint two point boundary condition. More sophisticated examples and applications to quantum systems are explored in 9]. We thank E. Prodan for suggesting the example below.

Let $q(x)$ be a real valued, continuous, even function on $[-1,1]$ and let $\alpha$ be a nonzero complex number satisfying $|\alpha|<1$. For a small parameter $\epsilon>0$, we define the operator

$$
\left[T_{\alpha} f\right](x)=-i f^{\prime}(x)+\epsilon q(x) f(x),
$$

with domain

$$
\mathcal{D}\left(T_{\alpha}\right)=\left\{f \in L^{2}[-1,1]: f^{\prime} \in L^{2}[-1,1], f(1)=\alpha f(-1)\right\} .
$$

Clearly $T_{\alpha}$ is a closed operator and $\mathcal{D}\left(T_{\alpha}\right)$ is dense in $L^{2}[-1,1]$.

If $C$ denotes the conjugation $[C u](x)=\overline{u(-x)}$ on $L^{2}[-1,1]$, then it follows that that nonselfadjoint operator $T_{\alpha}$ satisfies $T_{\alpha}=C T_{1 / \bar{\alpha}} C$. A short computation shows that $T_{\alpha}^{*}=T_{1 / \bar{\alpha}}$ and hence $T_{\alpha}$ is a $C$-selfadjoint operator.

In the case $\epsilon=0$, we have $T_{\alpha} f=-i f^{\prime}$ and we can explicitly compute the resolvent $R_{\alpha}$ of $T_{\alpha}$ at $z=0$ :

$$
\left[R_{\alpha}^{-1} f\right](x)=i \int_{-1}^{x} f(t) d t+\frac{i}{\alpha-1} \int_{-1}^{1} f(t) d t
$$


for $f$ in $L^{2}[-1,1]$. In particular, $\mathcal{D}\left(T_{\alpha}\right)=R_{\alpha}^{-1} L^{2}[-1,1]$ and $T_{\alpha} R_{\alpha}=I$. According to Theorem 7 , the antilinear problem

$$
-i f^{\prime}(x)=\sigma \overline{f(-x)}, \quad f \in \mathcal{D}\left(T_{\alpha}\right),
$$

admits nontrivial solutions for certain positive $\sigma_{n}$ tending to $\infty$. Moreover, the solutions $u_{0}, u_{1}, \ldots$ can be chosen to form a complete orthonormal system in $L^{2}[-1,1]$.

Taking another derivative in (27) and using back-substitution (see also Example 6), we find that the $u_{n}$ are solutions to $f^{\prime \prime}+\sigma_{n}^{2} f=0$, and thus

$$
u_{n}(x)=a_{n} e^{i \sigma_{n} x}+b_{n} e^{-i \sigma_{n} x}
$$

for certain constants $a_{n}$ and $b_{n}$. The boundary condition $u_{n}(1)=\alpha u_{n}(-1)$ shows that $a_{n} b_{n} \neq 0$ for all $n$.

Returning to the original first order antilinear equation (27), we see that

$$
\sigma_{n} a_{n} e^{i \sigma_{n} x}-\sigma_{n} b_{n} e^{-i \sigma_{n} x}=\sigma_{n} \overline{a_{n}} e^{i \sigma_{n} x}+\sigma_{n} \overline{b_{n}} e^{-i \sigma_{n} x},
$$

whence $a_{n}=\overline{a_{n}}$ and $b_{n}=-\overline{b_{n}}$. Multiplying $u_{n}$ by a suitable real constant, we obtain the (nonnormalized) eigenfunctions

$$
U_{n}(x)=e^{i \sigma_{n} x}+i \gamma_{n} e^{-i \sigma_{n} x},
$$

where $\gamma_{n}$ belongs to $\mathbb{R} \backslash\{0\}$. Moreover, the boundary condition $U_{n}(1)=\alpha U_{n}(-1)$ yields the equation

$$
e^{i \sigma_{n}}+i \gamma_{n} e^{-i \sigma_{n}}=\alpha\left[e^{-i \sigma_{n}}+i \gamma_{n} e^{i \sigma_{n}}\right]
$$

which implies that

$$
e^{2 i \sigma_{n}}=\frac{\alpha-i \gamma_{n}}{1-i \gamma_{n} \alpha}
$$

The image of the real line under the linear fractional transformation

$$
G(z)=\frac{\alpha-i z}{1-i z \alpha}
$$

is either a circle or a line which intersects the unit circle at exactly two points since $|G(0)|=|\alpha|<1$ and $|G(\infty)|=|1 / \alpha|>1$. In fact, the solutions $\gamma_{0}$ and $\gamma_{1}$ to $|G(z)|=1$ can be given in closed form:

$$
\frac{2 \operatorname{Im} \alpha \pm \sqrt{1-2 \operatorname{Re} \alpha^{2}+|\alpha|^{2}}}{1-|\alpha|^{2}}
$$

We may assume, after a possible relabeling, that the principal arguments $\sigma_{0}$ and $\sigma_{1}$ satisfying $e^{2 i \sigma_{0}}=G\left(\gamma_{0}\right)$ and $e^{2 i \sigma_{1}}=G\left(\gamma_{1}\right)$ satisfy $0<\sigma_{0}<\sigma_{1}<\pi$.

Retracing our steps, we have:

$$
\begin{aligned}
\sigma_{2 n} & =\sigma_{0}+n \pi, \\
\sigma_{2 n+1} & =\sigma_{1}+n \pi
\end{aligned}
$$

for $n \geq 0$. The associated (nonnormalized) eigenfunctions are:

$$
\begin{aligned}
U_{2 n}(x) & =e^{i\left(\sigma_{0}+n \pi\right) x}+i \gamma_{0} e^{-i\left(\sigma_{0}+n \pi\right) x}, \\
U_{2 n+1}(x) & =e^{i\left(\sigma_{1}+n \pi\right) x}+i \gamma_{1} e^{-i\left(\sigma_{1}+n \pi\right) x} .
\end{aligned}
$$

Using Corollary [6, we obtain the norm of the resolvent at $z=0$ :

$$
\left\|R_{\alpha}\right\|=\frac{1}{\sigma_{0}} .
$$


A familiar argument in perturbation theory shows that for $\epsilon\|q\|_{\infty}<\left\|R_{\alpha}\right\|$ the original operator (26) still has $z=0$ in its resolvent, and that a similar antilinear spectral picture holds. For instance, an estimate of $\left\|T_{\alpha}^{-1}\right\|$ is easily within reach.

\section{ACKNOWLEDGMENTS}

We are much indebted to E. Prodan, D. Sarason, and H.S. Shapiro.

\section{REFERENCES}

[1] Akhiezer, N.I., Glazman, I.M., Theory of Linear Operators in Hilbert Space, Dover, New York, 1993. MR1255973 (94i:47001)

[2] Bergman, S., Schiffer, M., Kernel functions and conformal mapping, Composition Math. 8 (1951), 205-249. MR0039812 (12:602c)

[3] Cascaval, R., Gesztesy, F., J-selfadjointness of a class of Dirac-type operators, J. Math. Anal. Appl. 294 (2004),no. 1, 113-121. MR2059793 (2005d:47079)

[4] Clark, D.N., One dimensional perturbations of restricted shifts, J. Anal. Math. 25, (1972) 169-191. MR0301534 (46:692)

[5] Duren, P.L., Theory of $H^{p}$ Spaces, Pure and Appl. Math., Vol 38, Academic Press, New York, 1970. MR0268655 (42:3552)

[6] Galindo, A., On the existence of J-selfadjoint extensions of J-symmetric operators with adjoint, Comm. Pure Appl. Math., 15(1962), 423-425. MR0149305 (26:6795)

[7] Garcia, S.R., Conjugation and Clark operators, Contemp. Math., vol. 393, Amer. Math. Soc., Providence, RI, 2006, pp. 67-111. MR2198373

[8] Garcia, S.R., Putinar, M., Complex symmetric operators and applications, Trans. Amer. Math. Soc. 358 (2006), 1285-1315. MR2187654 (2006j:47036)

[9] Garcia, S.R., Prodan, E., Putinar, M., Norm estimates of complex symmetric operators applied to quantum systems, J. Phys. A 39 (2006), 389-400. MR2198968 (2006j:81064)

[10] Glazman, I. M., An analogue of the extension theory of hermitian operators and a nonsymmetric one-dimensional boundary-value problem on a half-axis, Dokl. Akad. Nauk SSSR 115(1957), 214-216. MR0091440 (19:969d)

[11] Glazman, I.M., Direct methods of the qualitative spectral theory of singular differential operators (Russian), Gos. Iz. Fiz.-Mat. Lit., Moscow, 1963. MR0185471 (32:2938)

[12] Godič, V.I., Lucenko, I.E., On the representation of a unitary operator as a product of two involutions, Uspehi Mat. Nauk 20 (1965), 64-65. MR0190750 (32:8160)

[13] Gohberg, I., Krein, M.G., Introduction to the Theory of Linear Nonselfadjoint Operators in Hilbert Space, Amer. Math. Soc., Providence, R.I., 1969. MR0246142 (39:7447)

[14] Halmos, P.R., A Hilbert Space Problem Book (Second Edition), Springer-Verlag, 1982. MR0675952 (84e:47001)

[15] Keldysh, M.V., Lidskii, V.B., Questions of spectral theory of non-selfadjoint operators (in Russian) , Trudy IV-th. Soviet Math. Soc., Leningrad, Acad. Sci. SSSR Publ. House., 1963, 101-120. MR.0171183 (30:1414)

[16] Knowles, I., On the boundary conditions characterizing J-selfadjoint extensions of $J$ symmetric operators, J. Diff. Equations 40(1981), 193-216. MR0619134 (82i:47052)

[17] Koosis, P., Introduction to $H_{p}$ spaces (Second edition), Cambridge University Press, Cambridge, 1998. MR.1669574 (2000b:30052)

[18] Nikol'skiǔ, N.K., Treatise on the Shift Operator, Springer-Verlag, Berlin, 1986. MR0827223 (87i:47042)

[19] Poltoratski, A., Sarason, D., "Aleksandrov-Clark measures", Recent advances in operatorrelated function theory 1-14, Contemp. Math. vol. 393, Amer. Math. Soc., Providence, RI, 2006. MR2198367

[20] Race, D., The theory of J-selfadjoint extensions of J-symmetric operators, J. Diff. Equations 57(1985), 258-274. MR0788280 (86i:47031)

[21] Reed, M., Simon, B., Methods of Modern Mathematical Physics IV: Analysis of Operators, Academic Press, New York, 1978. MR 0493421 (58:12429c)

[22] Richter, S., Sundberg, C., A formula for the local Dirichlet integral, Michigan Math. J. 38 (1991), 355-379. MR1116495 (92i:47035) 
[23] Riss, U.V., Extension of the Hilbert space by J-unitary transformations, Helv. Phys. Acta 71(1998), 288-313. MR 1625826 (99e:47041)

[24] Schiffer, M., Fredholm eigenvalues and Grunsky matrices, Ann. Polonici Math. 39 (1981), 149- 164. MR0617457 (82k:30010)

[25] Takagi, T., On an algebraic problem related to an analytic theorem of Caratheodory and Fejer and on an allied theorem of Landau, Japan J. Math. 1 (1925), 83-93.

[26] Zhikhar, N.A., The theory of extensions of J-symmetric operators, Ukrainian Mat. Z. 11(1959), 352-364.

Department of Mathematics, University of California at Santa Barbara, Santa BarBARA, CALIFORNia 93106-3080

E-mail address: garcias@math.ucsb.edu

URL: http://math.ucsb.edu/ garcias

Current address: Department of Mathematics, Pomona College, Claremont, California 91711

E-mail address: Stephan.Garcia@pomona.edu

$U R L:$ http://pages.pomona.edu/ sg064747

Department of Mathematics, University of California at Santa Barbara, Santa Barbara, California 93106-3080

E-mail address: mputinar@math.ucsb.edu

$U R L:$ http://math.ucsb.edu/ mputinar 